\title{
COMPOSIÇÃo QUíMICA E ATIVIDADE BIOLÓGICA DAS FOLHAS E FRUTOS DE Triphasia trifolia
}

Renata P. dos Santos, Maria Teresa Salles Trevisan, Edilberto R. Silveira e Otília Deusdênia L. Pessoa*

Departamento de Química Orgânica e Inorgânica, Universidade Federal do Ceará, CP 12200, 60021-970 Fortaleza - CE, Brasil Vânia Maria M. Melo

Departamento de Biologia, Universidade Federal do Ceará, 60455-970 Fortaleza - CE, Brasil

Recebido em 10/11/06; aceito em 22/6/07; publicado na web em 19/12/07

\begin{abstract}
CHEMICAL COMPOSITION AND BIOLOGICAL ACTIVITY OF LEAVES AND FRUITS OF TRIPHASIA TRIFOLIA. The chemical composition of the essential oils from leaves and fruits of Triphasia trifolia was analyzed by GC-FID and GC-MS. The major constituents of oil obtained from leaves were sabinene (35.4\%) and myrcene (34.1\%), while the prevalent compounds in oil from fruits were sabinene (37.2\%), $\beta$-pinene (23.95) and $\gamma$-terpinene (16.3\%). Both oils showed moderate antimicrobial activity. The fruit decoction was also investigated leading to the isolation of the coumarins isopimpinelin, $(R)$-byakangelicin and $(S)$-mexoticin. From leaves were isolated the coumarins $(R)$-byakangelicin, aurapten, $(S)$-mexoticin, isosibiricin, isomerazin and coumurrayin and the flavonoid vitexin. All coumarins showed cholinesterase inhibition on TLC tests.
\end{abstract}

Keywords: Triphasia trifolia; coumarins; acetylcholinesterase activity.

\section{INTRODUÇÃO}

O gênero Triphasia (família: Rutaceae, tribo: Citreae, subtribo: Triphasiinae) restringe-se a apenas três espécies. O termo Triphasia origina-se do grego, onde "tripha" significa triplo, referindo-se à disposição das folhas. T. trifolia (Burm. f.) P. Wils., também conhecida pelas sinonímias $T$. trifoliata, T. aurantiola e Limonia trifolia, é um arbusto de origem asiática, que devido suas características ornamentais foi introduzido em vários países, especialmente naqueles de clima tropical, onde melhor se adapta ${ }^{1}$. Como consequiência da ampla ramificação e dos muitos espinhos, pontiagudos e resistentes, a planta é largamente cultivada em jardins, especialmente aos pés de muros, constituindo verdadeiras cercas vivas intransponíveis. Todas as suas partes são aromáticas e a fragrância exalada é agradável e típica dos citros. Os frutos, por exemplo, quando macerados exalam o aroma do limão, enquanto as folhas liberam o aroma da laranja. Em medicina popular, a infusão das folhas é empregada como vermicida ${ }^{2}$. T. trifolia tem sido objeto de várias investigações fitoquímicas, revelando ser uma rica fonte de cumarinas, particularmente de cumarinas preniladas ${ }^{2,3}$. Alcalóides ${ }^{2}$ e carotenóides ${ }^{4-6}$ também têm sido isolados a partir da espécie. Como é uma planta rica em glândulas oleíferas e de aspecto ornamental, a composição química dos óleos essenciais de plantas de diferentes origens tem sido analisada ${ }^{7,8}$.

Assim, como parte de um programa de pesquisa rotineiro sobre os óleos essenciais de plantas aromáticas da flora nordestina, nativas ou introduzidas, investigou-se a composição química dos óleos essenciais das folhas e frutos de T. trifolia, bem como suas propriedades antimicrobianas frente a uma série de bactérias. Adicionalmente, foram também investigados o decocto oriundo do processo de extração do óleo essencial dos frutos e os extratos das folhas de $T$. trifolia. Trevisan e Macedo ${ }^{9}$ relataram uma série de plantas com atividade anticolinesterase, que foi determinada através da avaliação dos extratos. Entre as plantas citadas, encontra-se T. trifolia, cujos extratos das folhas inibiram, em CCD, a enzima acetilcolinesterase. Em virtude destes resultados, todas as cumarinas iso-

*e-mail: opessoa@ufc.br ladas neste trabalho tiveram suas atividades avaliadas.

\section{RESULTADOS E DISCUSSÃO}

Os rendimentos dos óleos, calculados sobre a massa do material fresco, foram de $0,09 \%$ para as folhas e $0,07 \%$ para os frutos. Os componentes químicos identificados em ambos os óleos, incluindo seus respectivos índices de Kovat e porcentagens, estão sumarizados na Tabela 1. Um elevado percentual da composição química dos óleos foi determinado $(99,9 \%$ para as folhas e $98,4 \%$ para os frutos). O óleo essencial das folhas revelou ser constituído majoritariamente por monoterpenos $(90,0 \%)$, dos quais sabineno $(35,4 \%)$ e mirceno $(34,1 \%)$ foram os principais componentes. Outros componentes identificados em teores significativos foram hidrato de sesquisabineno $(6,3 \%), \gamma$-terpineno $(5,7 \%)$, limoneno $(4,5 \%) \mathrm{e}$ terpinen-4-ol $(4,1 \%)$. Da mesma forma, no óleo essencial dos frutos prevaleceram os monoterpenos, os quais representaram 92,0\% do total do óleo. Os constituintes majoritários foram sabineno $(37,2 \%), \beta$-pineno $(23,9 \%)$ e $\gamma$-terpineno $(16,3 \%)$, seguidos por limoneno $(5,3 \%)$. Em ambos os óleos, uma pequena fração de sesquiterpenos, representando 6,0 a $8,2 \%$, foi identificada. Exceto pela presença do hidrato de sesquisabineno, o qual contribui com $6,3 \%$ do óleo das folhas e $2,8 \%$ do óleo dos frutos, nenhum outro sesquiterpeno foi identificado em teor significativo. Digno de nota foram as presenças dos aldeídos alifáticos $n$-dodecanal e $n$-decanal no óleo das folhas, mesmo, em pequenas proporções $(3,3 \%)$. Conforme levantamento bibliográfico, foram encontrados dois trabalhos relacionados com a composição química volátil das folhas de T. trifolia, nativas de Cuba e Mauritius ${ }^{7,8}$, o que torna possível uma discussão sobre o perfil aromático destas plantas. Os principais componentes do óleo de T. trifolia de Mauritius ${ }^{7}$ foram os monoterpenos terpinen-4-ol $(29,0 \%)$ e carvacrol $(14,2 \%)$, enquanto na amostra de óleo de $\mathrm{Cuba}^{8}$, o sesquiterpeno germacreno B foi o constituinte em maior proporção (16,3\%), acompanhado do monoterpeno $\beta$-pineno $(8,3 \%)$. Uma comparação entre a composição química dos três óleos revelou que exceto pela presença do hidrato de sesquisabineno, todos os demais constituintes detectados no óleo de T. trifolia do Ceará foram encontrados no óleo da 
Tabela 1. Composição química $(\%)$ dos óleos essenciais das folhas e frutos de Triphasia trifolia

\begin{tabular}{|c|c|c|c|c|c|}
\hline Constituintes $^{\text {a }}$ & $\mathrm{IK}^{\mathrm{b}}$ & Folhas ${ }^{c}$ & Frutos $^{\mathrm{c}}$ & Folhas $^{\mathrm{d}}$ & Frutos $^{\mathrm{d}}$ \\
\hline$\alpha$-tujeno & 932 & - & 0,7 & 1,0 & 1,2 \\
\hline$\alpha$-pineno & 938 & 2,5 & 3,3 & 2,9 & 3,5 \\
\hline sabineno & 975 & 35,4 & 37,2 & 33,8 & 22,8 \\
\hline$\beta$-pineno & 979 & - & 23,9 & 0,7 & 29,9 \\
\hline mirceno & 991 & 34,1 & 1,3 & 33,6 & 1,5 \\
\hline$\delta$-careno & 1016 & 1,0 & 1,1 & 2,1 & 0,9 \\
\hline$p$-cimeno & 1019 & 1,1 & - & 5,1 & 9,7 \\
\hline limoneno & 1029 & 4,5 & 5,3 & 4,3 & 5,0 \\
\hline$\gamma$-terpineno & 1059 & 5,7 & 16,3 & 6,4 & 12,9 \\
\hline terpinoleno & 1089 & - & - & - & 1,2 \\
\hline 4-terpineol & 1174 & 4,1 & 3,3 & 6,9 & 8,9 \\
\hline$n$-decanal & 1203 & 1,6 & - & 0,8 & - \\
\hline$n$-dodecanal & 1403 & 1,7 & - & 0,6 & - \\
\hline $\operatorname{trans}$-cariofileno & 1419 & 1,2 & 1,0 & 1,6 & 0,6 \\
\hline$\alpha$-bergamoteno & 1435 & - & 2,2 & - & 1,6 \\
\hline$\beta$-curcumeno & 1515 & 0,7 & - & - & - \\
\hline hidrato de sesquisabineno & 1551 & 6,3 & 2,8 & - & - \\
\hline Total identificado & & 99,9 & 98,4 & 99,8 & 99,7 \\
\hline
\end{tabular}

${ }^{\mathrm{a}}$ Constituintes químicos listados de acordo com a ordem de eluição em coluna DB-5. ${ }^{\mathrm{b}}$ Índice de Kovat corrigido. ${ }^{\mathrm{c}}$ Coleta realizada em janeiro de 2005. ${ }^{\text {d }}$ Coleta realizada em janeiro de 2006, mesmo local.

espécie originária de Cuba, entretanto, apenas o monoterpeno 4terpineol, foi encontrado no óleo analisado de Mauritius. É importante destacar que este foi o único constituinte comum às três amostras de óleos.

O potencial antimicrobiano dos óleos essenciais foi avaliado frente a um painel constituído de oito cepas de bactérias, conforme disposto na Tabela 2. O óleo dos frutos mostrou-se ativo apenas contra dois tipos de bactérias, Chromobacterium violaceum e Bacillus subtilis. Por outro lado, o óleo das folhas mostrou eficácia contra todas as cepas testadas, embora moderadamente. A análise comparativa da composição química dos óleos revelou discretas diferenças qualitativas e quantitativas. Por exemplo, $p$-cimeno, decanal, dodecanal e $\beta$-curcumeno foram detectados apenas no óleo das folhas, entretanto, estes constituintes somam apenas $5,1 \%$ do total da constituição química daquele óleo. Deve-se notar que o monoterpeno mirceno, presente em $34,1 \%$ no óleo das folhas, no óleo dos frutos está presente em apenas 1,3\%. Desta forma, considerando que todos os demais constituintes do óleo das folhas são comuns ao óleo dos frutos, pode-se presumir que o mirceno tenha um importante papel na atividade expressada pelo óleo das folhas, sem, entretanto, desprezar uma possível ação sinergística provocada pela associação de alguns componentes químicos.

A prospecção química do decocto, oriundo do processo de hidrodestilação dos frutos de T. trifolia, resultou no isolamento de três cumarinas, cujas estruturas foram determinadas como sendo isopimpinelina $(\mathbf{1})^{10},(R)$-byakangelicina $(\mathbf{2})^{11,12}$ e $(S)$-mexoticina $(3)^{12,13}$. Posteriormente, deu-se início à investigação química dos extratos das folhas, tendo culminado no isolamento das cumarinas 2, 3, aurapteno $(\mathbf{4})^{14}$, coumurrayina $(\mathbf{5})^{15}$, isosibiricina $(\mathbf{6})^{13,16} \mathrm{e}$ isomerazina $(\mathbf{7})^{2}$, e do flavonóide vitexina $(\mathbf{8})^{17}$. As estruturas dos metabólitos secundários $\mathbf{1}$ - 8 foram determinadas por $\mathrm{RMN}{ }^{1} \mathrm{H}$ e ${ }^{13} \mathrm{C}$, uni- e bidimensional. Suas estruturas foram ainda confirmadas através da comparação de seus dados físicos e espectroscópicos com aqueles registrados na literatura. As cumarinas $\mathbf{1}-\mathbf{4}$ e $\mathbf{7}$ já haviam sido isolados de T. trifolia $^{2,12}$, mas as coumurrayina (5) e isosibiricina (6) e o flavonóide vitexina (8) estão sendo registrados pela primeira vez para a espécie. As Tabelas 3 e 4 apresentam num conjunto único, o resumo dos dados de $\mathrm{RMN}{ }^{1} \mathrm{H}$ e ${ }^{13} \mathrm{C}$ de todas as cumarinas (Figura 1) isoladas neste trabalho, relatadas erraticamente na literatura.

A atividade anticolinesterase apresentada pelos extratos das folhas de T. trifolia motivou a investigação destes extratos, visando o isolamento de metabólitos secundários responsáveis por tal atividade. Deste modo, todas as cumarinas isoladas de T. trifolia foram avaliadas em $\mathrm{CCD}$, frente à enzima acetilcolinesterase. $\mathrm{O}$ resultado deste bioensaio encontra-se descrito na Tabela 5 e, como pode ser observado, todos os compostos mostraram atividade.

Tabela 2. Atividade antibacteriana dos óleos essenciais das folhas e frutos de Triphasia trifolia

Média dos halos de inibição $(\mathrm{mm})^{\mathrm{a}}$

\begin{tabular}{lccc} 
Bactérias Gram-negativas & Folhas & Frutos & Tobramicina \\
\hline $\begin{array}{l}\text { Chromobacterium violaceum } \\
\text { (ATCC 12472) }\end{array}$ & 13,3 & 11,0 & 22,0 \\
$\begin{array}{l}\text { Enterobacter aerogenesis } \\
\text { (ATCC 13048) }\end{array}$ & 8,3 & $-{ }^{\mathrm{c}}$ & 17,0 \\
$\begin{array}{l}\text { Klebsiella pneumoniae } \\
\text { (ATCC 10031) }\end{array}$ & 8,0 & - & 18,0 \\
$\begin{array}{l}\text { Pseudomonas aeruginosa } \\
\text { (ATCC 25319) }\end{array}$ & 7,0 & - & 34,0 \\
$\begin{array}{l}\text { Salmonella choleraeasuis } \\
\text { (ATCC10708) }\end{array}$ & 8,6 & - & 22,0
\end{tabular}

\begin{tabular}{lccc}
\hline Bactérias Gram-positivas & & & \\
\hline $\begin{array}{l}\text { Bacillus subtilis } \\
\text { (ATCC 6633) }\end{array}$ & 9,0 & 9,5 & 31,0 \\
$\begin{array}{l}\text { Staphylococcus aureus } \\
\text { (ATCC 6538P) }\end{array}$ & 11,3 & - & 25,0 \\
$\begin{array}{l}\text { Staphylococcus aureus } \\
\text { (ATCC 25923) }\end{array}$ & 12,0 & - & 25,0 \\
\hline
\end{tabular}

a Os resultados expressam a média de 3 experimentos. ${ }^{\mathrm{b}}$ ATCC, "American Type Culture Collection". c (-) = Ausência de halo de inibição na dose testada. ${ }^{\mathrm{d}}$ Concentração dos óleos $=60 \mu \mathrm{g}$, do antibiótico $=10 \mu \mathrm{g}$. 
Tabela 3. Deslocamentos químicos de $\mathrm{RMN}{ }^{1} \mathrm{H}(500 \mathrm{MHz})$ das cumarinas $\mathbf{1}-\mathbf{7}$ isoladas de T. trifolia

\begin{tabular}{|c|c|c|c|c|c|c|c|}
\hline $\mathrm{H}$ & 1 & 2 & 3 & 4 & 5 & 6 & 7 \\
\hline 3 & $6,29(\mathrm{~d}, 9,8)$ & $6,27(\mathrm{~d}, 9,7)$ & $6,11(\mathrm{~d}, 9,6)$ & $6,20(\mathrm{~d}, 9,4)$ & $6,13(\mathrm{~d}, 9,5)$ & $6,12(\mathrm{~d}, 9,6)$ & $6,12(d, 9,4)$ \\
\hline 4 & $8,12(\mathrm{~d}, 9,8)$ & $8,21(\mathrm{~d}, 9,7)$ & $8,10(\mathrm{~d}, 9,6)$ & $7,60(\mathrm{~d}, 9,4)$ & $7,97(\mathrm{~d}, 9,5)$ & $7,97(\mathrm{~d}, 9,6)$ & $7,55(\mathrm{~d}, 9,4)$ \\
\hline 5 & - & - & - & $7,32(\mathrm{~d}, 8,6)$ & - & - & $7,29(\mathrm{~d}, 8,6)$ \\
\hline 6 & - & - & $5,59(\mathrm{~s})$ & $6,80(\mathrm{dd}, 2,4$ e 8,6$)$ & $6,32(s)$ & $6,33(\mathrm{~s})$ & $6,77(d, 8,6)$ \\
\hline 8 & - & - & - & $6,78(\mathrm{~d}, 2,4)$ & - & - & - \\
\hline $2^{\prime}$ & $7,63(\mathrm{~d}, 2,3)$ & $7,81(\mathrm{~d}, 2,3)$ & - & - & - & - & - \\
\hline $3^{\prime}$ & $7,00(\mathrm{~d}, 2,3)$ & $7,21(\mathrm{~d}, 2,3)$ & - & - & - & - & - \\
\hline $1 "$ & - & $\begin{array}{c}4,27 \\
(\mathrm{dd}, 10,3 \text { e } 8,0, \mathrm{Hb}) \\
4,55 \\
(\mathrm{dd}, 10,3 \text { e } 2,7, \mathrm{Ha})\end{array}$ & $2,93(\mathrm{~d}, 5,9)$ & $4,57(\mathrm{~d}, 6,5)$ & $3,44(\mathrm{~d}, 7,2)$ & $3,91(\mathrm{~s})$ & $3,93(\mathrm{~s})$ \\
\hline $2 "$ & - & $\begin{array}{c}3,82 \\
(\mathrm{dd}, 8,0 \text { e } 2,7)\end{array}$ & $\begin{array}{c}3,6 \\
(\mathrm{dd}, 7,0 \text { e } 6,0)\end{array}$ & $5,44(\mathrm{dt}, 1,0$ e 6,5$)$ & $5,20(\mathrm{~m})$ & - & - \\
\hline $3 "$ & - & - & - & - & - & 2,79 (hept, 6,9) & 2,75 (hept, 7,0) \\
\hline $4 "$ & - & $1,22(\mathrm{~s})$ & $1,27(\mathrm{~s})$ & $2,08(\mathrm{~m})$ & $1,66(\mathrm{~s})$ & $1,19(\mathrm{~d}, 6,9)$ & $1,14(\mathrm{~d}, 7,0)$ \\
\hline 5, & - & $1,27(\mathrm{~s})$ & $1,27(\mathrm{~s})$ & $2,08(\mathrm{~m})$ & $1,83(\mathrm{~s})$ & $1,19(\mathrm{~d}, 6,9)$ & $1,14(\mathrm{~d}, 7,0)$ \\
\hline $6 "$ & - & - & - & $5,06(\mathrm{~m})$ & - & - & - \\
\hline $8 "$ & - & - & - & $1,57(\mathrm{~s})$ & - & - & - \\
\hline $9 "$ & - & - & - & $1,64(s)$ & - & - & - \\
\hline $10 ”$ & - & - & - & $1,73(\mathrm{~s})$ & - & - & - \\
\hline $\mathrm{OCH}_{3}$ & $4,17(s)$ & 4,19 (s) & $3,95(\mathrm{~s})$ & - & $3,92(\mathrm{~s})$ & 3,92 (s) & 3,79 (s) \\
\hline $\mathrm{OCH}_{3}$ & $4,16(s)$ & - & $3,95(\mathrm{~s})$ & - & $3,92(\mathrm{~s})$ & $3,92(s)$ & - \\
\hline
\end{tabular}

Deslocamentos químcos $(\delta)$ em ppm. Constante de acoplamento (J) em Hz. Espectros obtidos em $\mathrm{CDCl}_{3}$.

Aurapteno (4) foi o mais ativo, com um halo de inibição de $8 \mathrm{~mm}$. Em seguida, isopimpinelina (1), $(R)$-byakangelicina $(\mathbf{2}) \mathrm{e}$ isomerazina (7) foram as cumarinas mais ativas, todas com halos de inibição igual a $7 \mathrm{~mm}$. Mexoticina (3) e isosibiricina (6) apresentaram halos de inibição de $6 \mathrm{~mm}$, enquanto coumurrayina (5) foi a cumarina com menor atividade. Estes dados reforçam o potencial anticolinesterásico das cumarinas e incluem plantas ricas

Tabela 4. Deslocamentos químicos de $\mathrm{RMN}{ }^{13} \mathrm{C}$ das cumarinas isoladas de Triphasia trifolia

\begin{tabular}{lccccccc}
\hline C & $\mathbf{1}$ & $\mathbf{2}$ & $\mathbf{3}$ & $\mathbf{4}$ & $\mathbf{5}$ & $\mathbf{6}$ & $\mathbf{7}$ \\
\hline 2 & 160,7 & 162,9 & 162,9 & 161,4 & 161,9 & 161,5 & 161,1 \\
3 & 113,0 & 113,2 & 109,4 & 112,9 & 110,9 & 111,1 & 113,1 \\
4 & 139,6 & 141,6 & 139,9 & 143,5 & 138,9 & 138,9 & 143,8 \\
$4 \mathrm{a}$ & 107,8 & 108,8 & 103,4 & 112,5 & 104,0 & 104,1 & 112,9 \\
5 & 144,5 & 146,2 & 156,1 & 128,7 & 155,4 & 156,5 & 127,5 \\
6 & 115,0 & 116,4 & 90,9 & 113,3 & 90,6 & 90,6 & 107,3 \\
7 & 150,2 & 151,8 & 162,4 & 162,2 & 161,1 & 161,4 & 160,5 \\
8 & 128,4 & 128,5 & 107,9 & 101,6 & 110,4 & 104,5 & 111,9 \\
$8 \mathrm{a}$ & 143,9 & 145,0 & 154,2 & 155,9 & 153,7 & 154,2 & 153,2 \\
2 & 145,3 & 147,1 & - & - & - & - & - \\
3, & 105,3 & 106,5 & - & - & - & - & - \\
$1 "$ & - & 76,9 & 24,2 & 65,5 & 21,6 & 34,5 & 34,7 \\
$2 "$ & - & 78,4 & 77,4 & 118,4 & 122,0 & 211,3 & 210,9 \\
$3 "$ & - & 72,9 & 72,7 & 142,4 & 132,3 & 40,9 & 40,9 \\
$4 "$ & - & 25,3 & 24,2 & 39,5 & 25,9 & 18,6 & 18,5 \\
5, & - & 23,9 & 24,2 & 26,3 & 18,1 & 18,6 & 18,5 \\
$6 "$ & - & - & - & 123,6 & & - & - \\
$7 "$ & - & - & - & 132,0 & & - & - \\
$8 "$ & - & - & - & 25,7 & & - & - \\
$9 "$ & - & - & - & 17,7 & & - & - \\
$10 "$ & - & - & - & 16,8 & & - & - \\
OCH $_{3}$ & 61,9 & 61,6 & 55,4 & - & 56,1 & 56,2 & 56,2 \\
OCH $_{3}$ & 61,0 & - & 55,4 & - & 56,2 & 56,3 & - \\
\hline & & & & & & &
\end{tabular}

neste tipo de compostos como promissoras candidatas à elaboração de drogas naturais que possam vir a auxiliar no tratamento da doença de Alzheimer. Na verdade, vários artigos têm sido publicados relatando a importância das cumarinas como agentes naturais anticolinesterásicos ${ }^{18,19}$.

\section{PARTE EXPERIMENTAL}

\section{Material vegetal}

As folhas e frutos de T. triflora foram coletados em janeiro de 2005, na praia de Icaraí, litoral oeste do Estado do Ceará. A autenticação da planta foi realizada pelo Prof. E. P. Nunes do Departamento de Biologia, UFC. Uma exsicata (№ 27245) da planta encontra-se depositada no Herbário Prisco Bezerra (EAC) da Universidade Federal do Ceará.

\section{Extração dos óleos}

Amostras, recém coletadas, de folhas $(620 \mathrm{~g})$ e frutos completamente maduros (900 g) foram submetidas a hidrodestilação por um período de $2 \mathrm{~h}$, utilizando doseador tipo-Clevenger. Os óleos essenciais extraídos foram secos com sulfato de sódio anidro, acondicionados em pequenos frascos de vidro e mantidos sob refrigeração antes de serem analisados.

\section{Análises dos óleos}

\section{$C G-E M$}

A análise qualitativa dos óleos foi realizada em espectrômetro Hewlett-Packard, modelo HP-5971 A, acoplado a cromatógrafo gáslíquido, modelo HP-5890 A, série II, equipado de coluna capilar apolar DB-5 (30 m x 0,25 mm x 0,25 $\mu \mathrm{m}$ ). Condições experimentais: ionização por impacto de elétrons a $70 \mathrm{eV}$; injetor no modo split (1:6); hélio como gás de arraste e fluxo de $1 \mathrm{~mL} / \mathrm{min}$; gradiente 
<smiles>[R]c1c2ccoc2c([R])c2oc(=O)ccc12</smiles>

$$
\mathrm{R}_{2}
$$$$
\mathrm{R}_{1}
$$

1<smiles>C1CCOC1</smiles>

$-\mathrm{OCH}_{3}$

2<smiles>CO[CH-][C@@H](O)C(O)([18OH])[18F]</smiles><smiles>[R]c1cc([R])c2oc(=O)ccc2c1[R]</smiles>

$\mathrm{R}_{1}$

$\mathrm{R}_{2}$
3<smiles>COC</smiles>

4 $-\mathrm{OCH}_{3}$ $\mathrm{H}$<smiles>CCC=C(C)C</smiles>

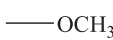

5 $-\mathrm{OCH}_{3}$

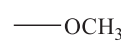

H
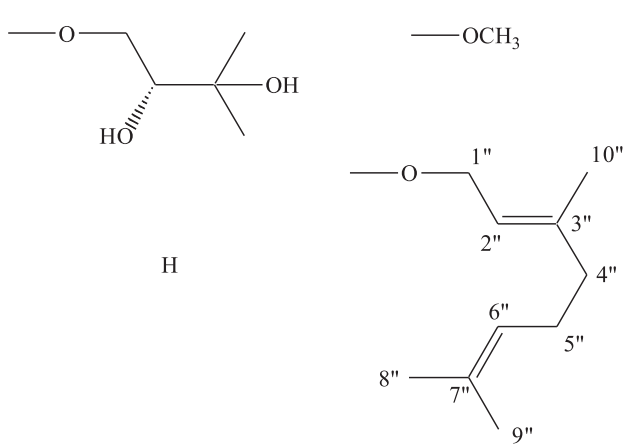

6 $-\mathrm{OCH}_{3}$<smiles>CCC(=O)C(C)C</smiles><smiles>CO[GeH2]</smiles><smiles>CCc1c(O)cc(O)c2c(=O)cc(-c3ccc(O)cc3)oc12</smiles>

Figura 1.

crescente de temperatura de $4{ }^{\circ} \mathrm{C} / \mathrm{min}$ de 50 a $180{ }^{\circ} \mathrm{C}$, e $20^{\circ} \mathrm{C} / \mathrm{min}$ de 180 a $280{ }^{\circ} \mathrm{C}$; temperatura do injetor $250{ }^{\circ} \mathrm{C}$ e a do detector $280{ }^{\circ} \mathrm{C}$. GC-FID:

A análise quantitativa dos óleos foi realizada em espectrômetro Shimadzu GC-17A, equipado com um detector de ionização de chama e coluna capilar apolar DB-5 (30 m x 0,25 mm x 0,25 $\mu \mathrm{m})$. Condições experimentais: injetor no modo split (1:5); hidrogênio como gás de arraste e fluxo de $1 \mathrm{~mL} / \mathrm{min}$; gradiente crescente de temperatura de $4{ }^{\circ} \mathrm{C} / \mathrm{min}$ de 35 a $180{ }^{\circ} \mathrm{C}$, e $17{ }^{\circ} \mathrm{C} / \mathrm{min}$ até atingir $280{ }^{\circ} \mathrm{C}$ e mantida nesta temperatura por $10 \mathrm{~min}$. As temperaturas do injetor e do detector foram de $280{ }^{\circ} \mathrm{C}$.

\section{Identificação dos constituintes químicos dos óleos essenciais}

As análises qualitativa e quantitativa dos componentes voláteis foram realizadas por CG-EM e CG-DIC, respectivamente, enquanto a identificação de cada constituinte foi realizada comparando-se o espectro de massa de cada componente com espectros de massa armazenados em espectroteca Wiley e banco de dados digital ${ }^{20,21}$, bem como por comparação com espectros de massa disponíveis na literatura ${ }^{22,23}$, considerando rigorosamente o padrão de fragmentação, alem do índice de Kovat corrigido para cada constituinte.

\section{Isolamento dos compostos}

O decocto dos frutos $(1,2 \mathrm{~L})$, oriundo do processo de extração do óleo essencial por hidrodestilação, foi filtrado e submetido à partição com AcOEt (4 x 200 mL). O extrato AcOEt $(770$ mg), obtido após evaporação do solvente, foi submetido a uma coluna cromatográfica empregando gel de sílica como adsorvente e como eluente hexano/ $\mathrm{CH}_{2} \mathrm{Cl}_{2}$ (9:1 a 1:9), $\mathrm{CH}_{2} \mathrm{Cl}_{2}, \mathrm{CH}_{2} \mathrm{Cl}_{2} / \mathrm{AcOEt}$ (9:1, 7:3, 1:1, 3:7) e AcOEt. Foram coletadas um total de 48 frações de $50 \mathrm{~mL}$ que, após monitoramento por $\mathrm{CCD}$, foram reunidas em 10 frações $(\mathrm{F} 1$ - F10). A fração F-2, obtida por eluição com hexano/ $\mathrm{CH}_{2} \mathrm{Cl}_{2}$ 1:1, mostrou ser constituída por uma única substância (1; $5,5 \mathrm{mg}$ ). A fração F-8 (264 mg), obtida por eluição com $\mathrm{CH}_{2} \mathrm{Cl}_{2} /$ AcOEt 7:3, foi submetida à cromatografia em gel de sílica e eluída com $\mathrm{CH}_{2} \mathrm{Cl}_{2} / \mathrm{AcOEt}$ (9:1, 8:2, 7:3, 6:4) obtendo-se 105 frações de $10 \mathrm{~mL}$. Após monitoramento em CCD, as frações 79 a 100, obtidas por eluição com $\mathrm{CH}_{2} \mathrm{Cl}_{2} / \mathrm{AcOEt}$ 8:2 e 7:3, foram reunidas e o material resultante $(153 \mathrm{mg})$ foi sujeito à cromatografia, resultando no isolamento do composto $2(9,0 \mathrm{mg})$, por eluição com hexano/AcOEt 1:1. A fração F-9 (30,0 mg) foi submetida a uma cromatografia flash utilizando hexano/AcOEt 2:8 como eluente para fornecer o composto 3 (3,0 mg).

As folhas de $T$. trifolia, secas a temperatura ambiente e trituradas $(6,0 \mathrm{~kg})$, foram extraídas exaustivamente com éter de petróleo/ AcOEt 1:1 e em seguida com EtOH, resultando nos respectivos extratos, após evaporação dos solventes. O extrato éter de petróleo/AcOEt 1:1 (260 g) foi fracionado sobre gel de sílica resultando nas seguintes frações: hexano $(135,4 \mathrm{~g}), \mathrm{CH}_{2} \mathrm{Cl}_{2}(88,1 \mathrm{~g})$, AcOEt $(26,3 \mathrm{~g})$ e $\mathrm{MeOH}(10,1 \mathrm{~g})$. Uma alíquota da fração hexano (40 g) foi submetida à cromatografia em gel de sílica empregando os solventes éter de petróleo, hexano hexano/ $\mathrm{CH}_{2} \mathrm{Cl}_{2} 1: 1, \mathrm{CH}_{2} \mathrm{Cl}_{2}$, AcOEt e MeOH. A fração hexano/ $\mathrm{CH}_{2} \mathrm{Cl}_{2}$ 1:1 (14,0 g) foi submetida à cromatografia flash, utilizando como eluente hexano/AcOEt 8:2, obtendo-se 30 frações de $25 \mathrm{~mL}$. A fração 3/4, após recristalização em $\mathrm{MeOH}$, forneceu a substância 4 (198,2 mg). A fração 5/9 (1,0 g), após cromatografia flash, empregando hexano/ AcOEt 9:1, resultou no isolamento do composto 5 (12,4 mg). A fração $\mathrm{CH}_{2} \mathrm{Cl}_{2}(3,2 \mathrm{~g})$ foi submetida à cromatografia em gel de sílica e eluída com hexano/ $\mathrm{CH}_{2} \mathrm{Cl}_{2}(7: 3,1: 1,3: 7) \mathrm{CH}_{2} \mathrm{Cl}_{2}, \mathrm{CH}_{2} \mathrm{Cl}_{2} /$ AcOEt (7:3, 1:1, 3:7) e AcOEt. Após CCD, as frações obtidas por 
eluição com hexano/ $\mathrm{CH}_{2} \mathrm{Cl}_{2}(7: 3,1: 1)$ foram reunidas $(198 \mathrm{mg})$ e submetidas à cromatografia flash utilizando o eluente hexano/ AcOEt 7:3. Como resultado, foi isolado o composto 6 (14,3 mg), após recristalização em $\mathrm{MeOH}$. Parte do extrato EtOH das folhas de T. trifolia $(157 \mathrm{~g})$, foi suspendido em água e extraído com $\mathrm{CH}_{2} \mathrm{Cl}_{2}$, AcOEt e $n$-BuOH. A fração $\mathrm{CH}_{2} \mathrm{Cl}_{2}(33,4 \mathrm{~g})$ foi sujeita à cromatografia em gel de sílica empregando misturas de hexano/ AcOEt, cujo gradiente de polaridade foi aumentado gradativamente de 10\%, até atingir AcOEt. Na fração hexano/AcOEt 3:7, formouse um precipitado que após filtração resultou no composto 2 (30 mg). A fração obtida por eluição com hexano/AcOEt 6:4 (7 g) após cromatografia flash, usando o sistema de solvente hexano/ $\mathrm{CH}_{2} \mathrm{Cl}_{2} /$ AcOEt 5:3:2, forneceu o composto 7 (726 mg). A fração AcOEt $(3,4 \mathrm{~g})$ foi submetida à cromatografia em gel de sílica tendo como eluente misturas de hexano/AcOEt 7:3, 1:1, 3:7, AcOEt e MeOH. A subfração hexano/AcOEt 7:3 (1,28 g), após cromatografia flash e por eluição com AcOEt/ $\mathrm{CH}_{2} \mathrm{Cl}_{2} /$ hexano 6:2:2 resultou no isolamento de 3 (11,3 mg). A fração AcOEt (11,7 g), resultante da partição, foi submetida a uma rápida coluna cromatográfica empregando como eluentes misturas de $\mathrm{CH}_{2} \mathrm{Cl}_{2} / \mathrm{AcOEt}$ (8:2, 6:4, 4:6, 2:8), AcOEt e MeOH. Na fração AcOEt, formou-se um precipitado, cuja tentativa de purificação por recristalização foi inútil. Este material (250 mg), após cromatografia em gel de sílica utilizando como eluente $\mathrm{CH}_{2} \mathrm{Cl}_{2}$ /acetona $(1: 1,3: 7,2: 8)$, acetona e $\mathrm{MeOH}$, conduziu ao isolamento do composto 8 (35 mg), um sólido amorfo amarelo, obtido por eluição com a mistura binária $\mathrm{CH}_{2} \mathrm{Cl}_{2}$ /acetona (3:7).

\section{Ensaio antibacteriano}

Os óleos essenciais das folhas e frutos de T. triflolia foram diluídos em clorofórmio (40 mg/mL) e submetidos a ensaios de atividade antibacteriana pelo método de difusão em disco, como descrito por Bauer e colaboradores ${ }^{24}$. Para tanto, culturas de bactérias, provenientes da "American Type Culture Collection", em fase exponencial de crescimento $\left[10^{7}-10^{8}\right.$ Unidades Formadoras de Colônias (UFC)/mL] foram semeadas em placas de agar Müller-Hinton (Difco, USA), em duplicata, utilizando "swabs" estéreis. Discos de papel de filtro da Whatman AA (6 mm de diâmetro) esterilizados foram distribuídos em placas de Petri esterilizadas e separadamente embebidos com $15 \mu \mathrm{L}$ de cada óleo ou com $15 \mu \mathrm{L}$ de clorofórmio, controle negativo. Após completa evaporação do clorofórmio, os discos contendo $60 \mu \mathrm{g}$ de óleo foram colocados sobre as superfícies das placas previamente semeadas com as bactérias. As placas foram incubadas a $35^{\circ} \mathrm{C}$ por $24 \mathrm{~h}$ e então examinadas quanto à presença de zonas de inibição do crescimento. Discos do antibiótico comercial Tobramicina (10 $\mu \mathrm{g}$; Cecon, São Paulo) foram usados como controle positivo para todas as bactérias testadas.

\section{Ensaio da atividade inibitória da enzima acetilcolinesterase}

A inibição da enzima acetilcolinesterase (AChE), através de CCD, pôde ser avaliada seguindo a metodologia descrita por Elmann $^{25}$, a qual foi posteriormente adaptada por Rhee et al. ${ }^{26}$. Este bioensaio consiste na aplicação da amostra em CCD, seguida da pulverização da placa com o reagente de Ellman (ácido 5,5'-ditiobis[2-nitrobenzóico, DTNB) e uma solução de iodeto de acetiltiocolina (ATCI) em tampão. Após este procedimento, pulveriza-se a placa com a enzima AChE (3 U/mL). Decorridos alguns minutos ( 3), a inibição enzimática pode ser constatada pela ausência da cor amarela e concomitante surgimento de um halo branco. O ensaio enzimático em CCD é um teste puramente qualitativo, mas de sensibilidade significativa. Para a realização do teste foi necessária a preparação das seguintes soluções: (1) $50 \mathrm{mM}$ Tris/HCl pH 8 (so- lução tampão); (2) $50 \mathrm{mM}$ Tris/ $\mathrm{HCl} \mathrm{pH} 8$, contendo $0,1 \%$ de albumina sérica bovina (BSA) fração $\mathrm{V}$; (3) $1 \mathrm{mM}$ do reagente de Ellman e (4) $1 \mathrm{mM}$ de ACTI. A enzima AChE liofilizada foi dissolvida na solução tampão (1) para fazer uma solução estoque de 1000 U/mL. Para a diluição da enzima utilizou-se a solução tampão (2).

Inicialmente, alíquotas de $5 \mu \mathrm{L}$ dos compostos 1 a 7 , na concentração de $4 \mathrm{mg} / \mathrm{mL}$, foram aplicados em CCD (DC-Alufolien, Silicagel 60 F254, 0,2 mm Merck). Em seguida pulverizou-se a placa com as soluções (3) e (4). Após 3 min, tempo necessário para a completa secagem das soluções, pulverizou-se a placa com a enzima AChE $(3 \mathrm{U} / \mathrm{mL})$. Decorridos aproximadamente $10 \mathrm{~min}$, observou-se o desaparecimento da cor amarela e o aparecimento de halos brancos, cujos diâmetros foram imediatamente medidos. Como controle positivo foi usado o carbachol, conforme metodologia descrita por Rhee et al. ${ }^{26}$. O resultado deste ensaio encontra-se descrito na Tabela 5.

Tabela 5. Inibição da enzima acetilcolinesterase apresentada pelas cumarinas $\mathbf{1}$ - $\mathbf{7}$ isoladas de Triphasia trifolia

\begin{tabular}{lccc}
\hline Compostos & $\begin{array}{c}\text { Halos de } \\
\text { inibição }(\mathrm{mm})\end{array}$ & Compostos & $\begin{array}{c}\text { Halos de } \\
\text { inibição }(\mathrm{mm})\end{array}$ \\
\hline $\mathbf{1}$ & 7 & $\mathbf{5}$ & 5 \\
$\mathbf{2}$ & 7 & $\mathbf{6}$ & 6 \\
$\mathbf{3}$ & 6 & $\mathbf{7}$ & 7 \\
$\mathbf{4}$ & 8 & carbachol & 11 \\
\hline
\end{tabular}

Concentração $=4 \mathrm{mg} / \mathrm{mL}$

\section{CONCLUSÕES}

T. trifolia é uma planta exótica, cuja composição química volátil e não-volátil somente das folhas vem sendo investigada ao longo dos anos. Neste trabalho, além das folhas, investigou-se também a composição química dos frutos e, de acordo com os resultados obtidos, não só as folhas como os frutos constituem uma fonte de cumarinas e óleo essencial. Em adição às já conhecidas cumarinas, neste trabalho são isoladas duas cumarinas, coumurrayina e isosibiricina, além do flavonóide vitexina, ainda não registrados para a espécie. Os óleos essenciais de T. trifolia, particularmente o das folhas, apresentaram moderada atividade antimicrobiana, enquanto as cumarinas, num ensaio preliminar, foram capazes de inibir a enzima acetilcolinesterase. Estes resultados fazem de T. trifolia uma promissora fonte de agentes naturais anticolinesterásicos e, neste contexto, justificam futuros estudos (in vitro e in vivo) no sentido de prescrustar o potencial destes compostos como coadjuvantes no tratamento da doença de Alzheimer.

\section{AGRADECIMENTOS}

Às instituições de fomento à pesquisa CNPq, CAPES, FUNCAP e PRONEX pelo auxílio financeiro.

\section{REFERÊNCIAS}

1. Bailey, L. H.; Manual of Cultivated Plants: Most Commonly grown in the Continental United States and Canada, The Macmillan Company: New York, 1954.

2. Abaul, J.; Philogenese, E.; Bourgeois, P.; Poupat, C.; Ahond, A.; Potier, P.; J. Nat. Prod. 1994, 57, 846.

3. Da Silva, L. B.; Herath, W. H. M. W.; Jennings, R. C.; Mahendran, M.; Wannigama, G. P.; Phytochemistry 1981, 20, 2776.

4. Yokoyama, H.; White, M. J.; Phytochemistry 1970, 9, 1795.

5. Yokoyama, H.; Guerrero, H. C.; J. Org. Chem. 1970, 35, 2080.

6. Yokoyama, H.; White, M. J.; Phytochemistry 1968, 7, 1031.

7. Gurib-Fakin, A.; Sewraj, M. D.; Narod, F.; Menut, C.; J. Essent. Oil Res. 
$1995,7,215$.

8. Pino, J. A.; Marbot, R.; Fuents, V.; J. Essent. Oil Res. 2006, 18, 24

9. Trevisan, M. T. S.; Macedo, F. V. V.; Quim. Nova 2003, 26, 301.

10. Liu, R.; Feng, L.; Sun, A.; Kong, L.; J. Chromatogr., A 2004, 1055, 71.

11. Adebajo, A. C.; Reisch, J.; Fitoterapia 2000, 71, 334.

12. Ruangrungsi, N.; Lange, G. L.; Organ, M. G.; Fitoterapia 1994, 65, 179.

13. Imai, F.; Itoh, K.; Kishibuchi, N.; Kinoshita, T.; Sankawa, U.; Chem. Pharm. Bull. 1989, 1, 119.

14. Abulrob, A. N.; Suller, M. T. E.; Gumbleton, M.; Simons, C.; Russell, A. D.; Phytochemistry 2004, 65, 3021.

15. Wu, T. S.; Tien, H. J.; Arisawa, M.; Shimizu, M.; Morita, N.; Phytochemistry 1980, 19, 2227.

16. Macias, F. A.; Massanet, G. M.; Rodriguez-Luis, F.; Magn. Reson. Chem. 1989, 27, 892.

17. Palme, E.; Bilia, A. R.; Feo, V.; Morelli, I.; Phytochemistry 1994, 35, 1381.

18. Kang, S. Y.; Lee, K. Y.; Sung, S. H.; Park, M. J.; Kim, Y. C.; J. Nat. Prod. 2001, 64, 683
19. Brühlmann, C.; Ooms, F.; Carrupt, P. A.; Testa, B.; Catto, M.; Leonetti, F.; Altomare, C.; Carrotti, A.; J. Med. Chem. 2001, 44, 3195.

20. Craveiro, A. A.; Matos, F. J. A.; Alencar, J. W.; J. Nat. Prod. 1984, 47, 890.

21. Alencar, J. W.; Craveiro, A. A.; Matos, F. J. A.; Machado, M. I. L.; Quim. Nova 1990, 13, 282

22. Stenhagen, E.; Abrahamson S.; McLafferty, F. W.; Registry of Mass Spectra Data, J. Wiley \& Sons: New York, 1974.

23. Adams, R. P.; Identification of Essential Oil Components by Gas Chromatography/Quadrupole Mass Spectroscopy, Allured Publishing Corporation: Illinois, 2001.

24. Bauer, A. W.; Kirb, W. M. M.; Sherris, J. C.; Turck, M.; Am. J. Clinic Pathol. 1966, 45, 493

25. Ellman, G. L.; Biochem. Pharmacol. 1961, 7, 88.

26. Rhee, I. K.; Meent, M. V. D.; Ingkanina, K.; Verpoorte, R.; J. Chromatogr. 2001, 915, 217. 